\title{
Manipulation of fungal development as source of novel secondary metabolites for biotechnology
}

\author{
Jennifer Gerke • Gerhard H. Braus
}

Received: 27 May 2014 / Revised: 25 July 2014 / Accepted: 28 July 2014 / Published online: 21 August 2014

(C) The Author(s) 2014. This article is published with open access at Springerlink.com

\begin{abstract}
Fungal genomics revealed a large potential of yetunexplored secondary metabolites, which are not produced during vegetative growth. The discovery of novel bioactive compounds is increasingly gaining importance. The high number of resistances against established antibiotics requires novel drugs to counteract increasing human and animal mortality rates. In addition, growth of plant pathogens has to be controlled to minimize harvest losses. An additional critical issue is the post-harvest production of deleterious mycotoxins. Fungal development and secondary metabolite production are linked processes. Therefore, molecular regulators of development might be suitable to discover new bioactive fungal molecules or to serve as targets to control fungal growth, development, or secondary metabolite production. The fungal impact is relevant as well for our healthcare systems as for agriculture. We propose here to use the knowledge about mutant strains discovered in fungal model systems for a broader application to detect and explore new fungal drugs or toxins. As examples, mutant strains impaired in two conserved eukaryotic regulatory complexes are discussed. The COP9 signalosome (CSN) and the velvet complex act at the interface between development and secondary metabolism. The CSN is a multi-protein complex of up to eight subunits and controls the activation of CULLIN-RING E3 ubiquitin ligases, which mark substrates with ubiquitin chains for protein degradation by the proteasome. The nuclear velvet complex consists of the velvet-domain proteins VeA and VelB and the putative methyltransferase LaeA acting as a global regulator for secondary metabolism. Defects in both complexes disturb fungal development, light perception, and the control of secondary metabolism. The potential biotechnological
\end{abstract}

\footnotetext{
J. Gerke $\cdot$ G. H. Braus $(\bowtie)$

Institut für Mikrobiologie \& Genetik, Georg-August-Universität, Grisebachstr. 8, D-37077 Göttingen, Germany

e-mail: gbraus@gwdg.de
}

relevance of these developmental fungal mutant strains for drug discovery, agriculture, food safety, and human healthcare is discussed.

Keywords Velvet domain proteins · COP9 signalosome CSN $\cdot$ Secondary metabolites $\cdot$ Fungal development

\section{Introduction}

Fungi play an important role in our everyday life, whether positive or negative. On the one hand, they are origins of lifesaving and life-enhancing drugs, food additives, and aromas, but, on the other hand, they have the potential to contaminate our crops and food or to lead to serious infections. Fungi, as well as bacteria, plants, and some insects, produce secondary metabolites. These natural products are low-molecular-weight molecules that, unlike primary metabolites, are dispensable for survival of the organism but confer an advantage in specific habitats or during changes in environmental conditions.

Many secondary metabolites possess biological activities that can range from beneficial to harmful (Fig. 1). Beneficial secondary metabolites (SMs) include antibacterial agents such as penicillin, antifungal agents such as caspofungin, anticancer drugs such as taxol, immunosuppressive drugs such as ciclosporin, or cholesterol-lowering drugs such as lovastatin. Around $50 \%$ of the newly approved drugs between 1981 and 2010 were of secondary metabolite origin (Newman and Cragg 2012), emphasizing the high importance of studies in this biological field. A growing problem is the tremendous current and future increase in resistances against established antibiotics as was foretold by the WHO (Cooper and Shlaes 2011). The daily application especially of antibiotics in clinical medicine, stock breeding, and agriculture leads to the development of multi-resistances, where known antibiotics 


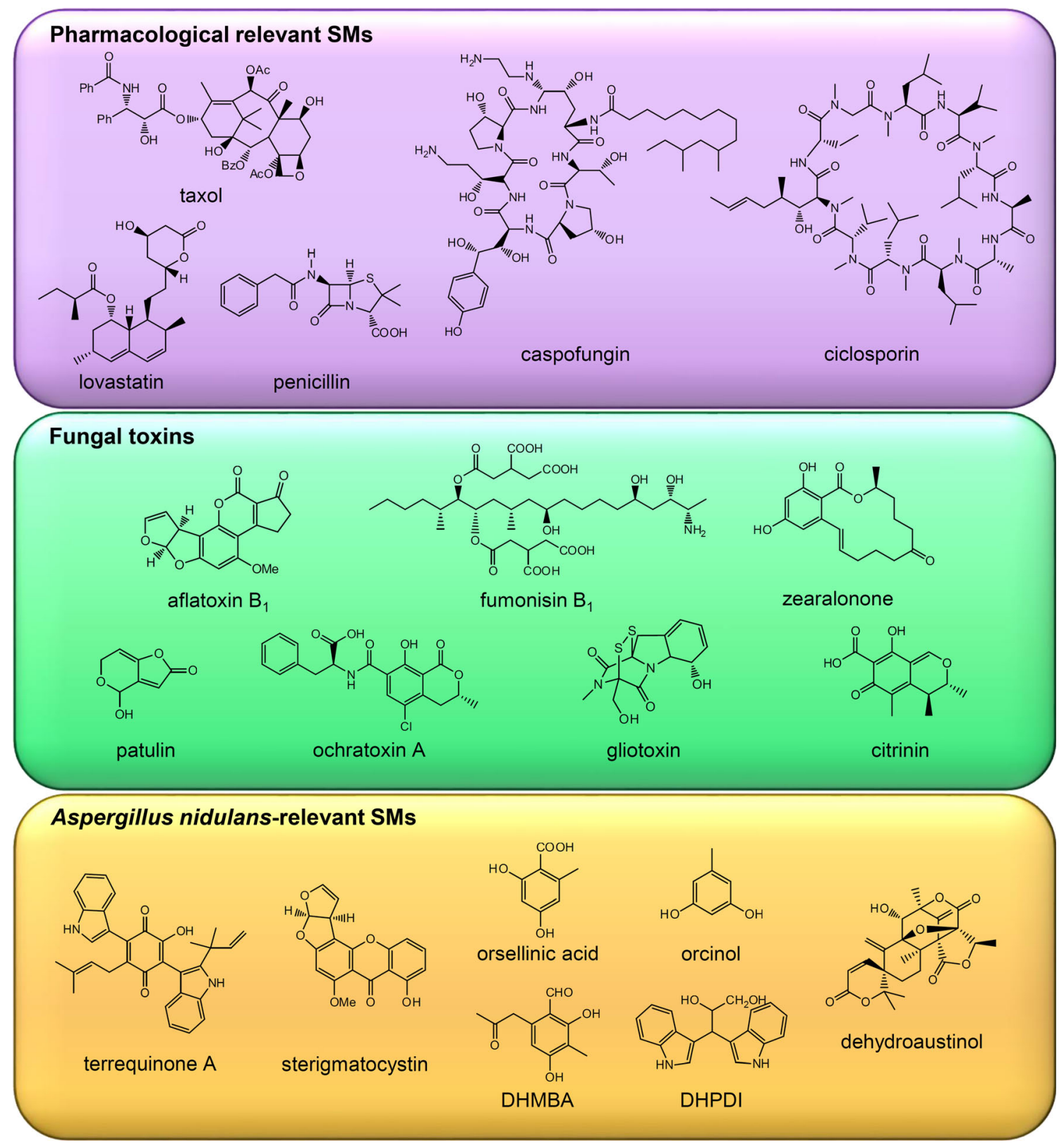

Fig. 1 Chemical structures of pharmacological relevant secondary metabolites (SMs), fungal toxins and A. nidulans-relevant SMs

are ineffective. Therefore, the discovery of novel drugs is imperative.

The progress in sequencing techniques allows deciphering genomes of more and more organisms within a very short time. The resulting data uncovered a significant genomic potential in many fungi to produce secondary metabolites (Inglis et al. 2013). The biosynthetic genes for the production of SMs in fungi are arranged in clusters. The expression of many of these gene clusters is silenced under standard laboratory conditions. One strategy to discover new bioactive secondary metabolites is the activation of silenced SM gene clusters (Brakhage 2013; Hertweck 2009; Lim et al. 2012). However, the expression of one gene cluster might affect the expression of other gene clusters, what complicates identification of the corresponding SM products (Bergmann et al. 2010; Gerke et al. 2012b; Wiemann et al. 2013).

Fungi are not only producers of drugs, but are also industrially exploited to produce enzymes or food additives. For example, Aspergillus niger is used for large-scale fermentation of citric acid and gluconic acid (Berovic and Legisa 2007; Singh and Kumar 2007). In Asian cuisine, Aspergillus oryzae is used for fermentation of soybeans, saccharification of rice, and production of alcoholic drinks and rice vinegars (Kobayashi et al. 2007). Monascus purpureus is used for natural food coloring (Went 1895). Due to the usage of fungi in food preparation, the knowledge of available secondary 
metabolite gene clusters becomes even more relevant, as potentially harmful gene clusters might 'doze' in the genome and represent an intoxication risk. Prominent examples of deleterious SMs are aflatoxins, a group of mycotoxins produced by several Aspergillus species, followed by citrinin and patulin, which are produced by Aspergillus and Penicillium species, and Fusarium-specific toxins such as zearalenone. The contamination of crops with mycotoxin-producing fungi leads to a worldwide yield loss of agricultural crops of more than $10 \%$ (Normile 2010), representing an enormous economic problem. However, fungal spores are not only a risk for plants, but can also pose serious health problems for humans. Inhalation of fungal spores can induce allergic reactions, and in the case of Aspergillus fumigatus, Aspergillus flavus, and Aspergillus terreus, an infection can lead to life-threatening invasive aspergillosis in immunocompromised patients.

A comprehensive understanding of genetic regulation is necessary to compete with nature and to handle the three topics: drug discovery, toxin contamination, and virulence. An interesting approach for biotechnology of yet-unexplored fungi might be to create and use mutations in conserved protein complexes at the interface between fungal development and secondary metabolism. This approach is exemplified here for two well-studied protein complexes: the COP9 signalosome (CSN) and the velvet complex.

\section{The COP9 signalosome complex CSN: control of fungal protein degradation}

\section{$\mathrm{CSN}$ and protein degradation}

CSN is a multi-protein complex consisting of up to eight subunits, which is conserved among eukaryotes. It was first identified in plants $(\mathrm{COP}=$ constitutively photo morphogenic/ de-etiolated/fusca), where mutated $\operatorname{csn}$ genes induce expression of light-regulated genes in darkness (Wei et al. 1994). In mammals and other higher eukaryotes, disruption of $\operatorname{csn}$ genes is lethal, whereas deletions in filamentous fungi and unicellular yeasts lead to viable mutants (Busch et al. 2003; Mundt et al. 2002). Neurospora crassa harbors only seven subunits, whereas in Schizosaccharomyces pombe only six subunits are conserved. The fact that all eight CSN subunits are conserved in Aspergillus nidulans (CsnA-CsnH) makes it an attractive model organism to study CSN function (Braus et al. 2010).

The CSN is structurally similar to the $26 \mathrm{~S}$ proteasome or the eukaryotic translation initiation factor eIF3, and the eight subunits consist of six PCI (proteasome/COP9/initiation factor) domain proteins and two MPN (Mprlp and Padlp Nterminal) domain proteins. The highly conserved fifth subunit CSN5/CsnE harbors the catalytic function of the complex, a JAMM motif with metalloprotease activity, and can recruit the whole CSN complex in the presence of the other subunits
(Busch et al. 2003, 2007; Cope et al. 2002). The main function of CSN is to control the activity of CULLIN-RING E3 ubiquitin ligases (CRLs), which ubiquitinate proteins and target them for degradation by the 26S proteasome (Braus et al. 2010; Wei et al. 2008). CRLs are composed of a cullin and a RING-Finger domain protein as a scaffold onto which the ubiquitin-conjugating enzyme E2 binds to transfer the ubiquitin to the substrate (Fig. 2). Additionally, the cullin binds to adaptors that recruit the target substrate. The largest group of CULLIN-RING E3 ubiquitin ligases are SCF (Skp1/çullin-1/ F-box) E3 ubiquitin ligases. They consist of a cullin-1 scaffold C-terminally bound to the RING-finger domain protein RBX1/RbxA and N-terminally bound to Skp1/SkpA, which bridges the complex to the substrate-recruiting F-box proteins. The SCF complex is activated by neddylation, which changes the conformation of the complex in such a way that the target substrate and the ubiquitin, which is delivered by the E2conjugating enzyme through the RING protein, are in sufficiently close proximity (Duda et al. 2008). Neddylation is a process where the small ubiquitin-like modifier Nedd8 is covalently attached to a lysine side chain of a cullin (Wu et al. 2005). This process is reversible by deneddylation. The deconjugation of Nedd8 inactivates cullin complexes. CSN functions as a deneddylating enzyme of cullins. The original finding that in vitro neddylation activates and deneddylation inactivates CULLIN-RING E3 ubiquitin ligases, whereas in vivo CRL activity requires deneddylation, is referred to as the COP9 signalosome paradox (Schmidt et al. 2009). In A. nidulans, around $70 \mathrm{~F}-$ box proteins are known to be responsible for recruiting different substrates. The activity of the SCF complex is regulated by cycles of disassembly and assembly corresponding to cycles in neddylation and deneddylation. These cycles are presumably necessary to replace F-box proteins which had been ubiquitinated due to the lack of substrates, or to replace F-box proteins which are no more required during developmental processes (Braus et al. 2010). However, CSN is not the only fungal deneddylase. DenA is a second deneddylase, which is conserved in eukaryotes and which physically interacts with CSN. The interaction of both deneddylases affects the half-life of the corresponding proteins (Christmann et al. 2013).

The neddylation and deneddylation cycles of CULLINRING E3 ubiquitin ligases require the cullin-associated Nedd8-dissociated protein CandA as an additional control factor (Helmstaedt et al. 2011). CandA stably binds to deneddylated cullins and by this inhibits CRL activity in vitro. In vivo, it was shown that CandA is needed to initiate the exchange of F-box proteins on SCFs (Lo and Hannink 2006). The binding of CandA to the cullin leads to disassembly of the SCF complex, and by a subsequent neddylation step, the complex can be reassembled with an exchanged Fbox protein. Taken together, CSN and CandA both activate CULLIN-RING E3 ubiquitin ligases in vivo. 


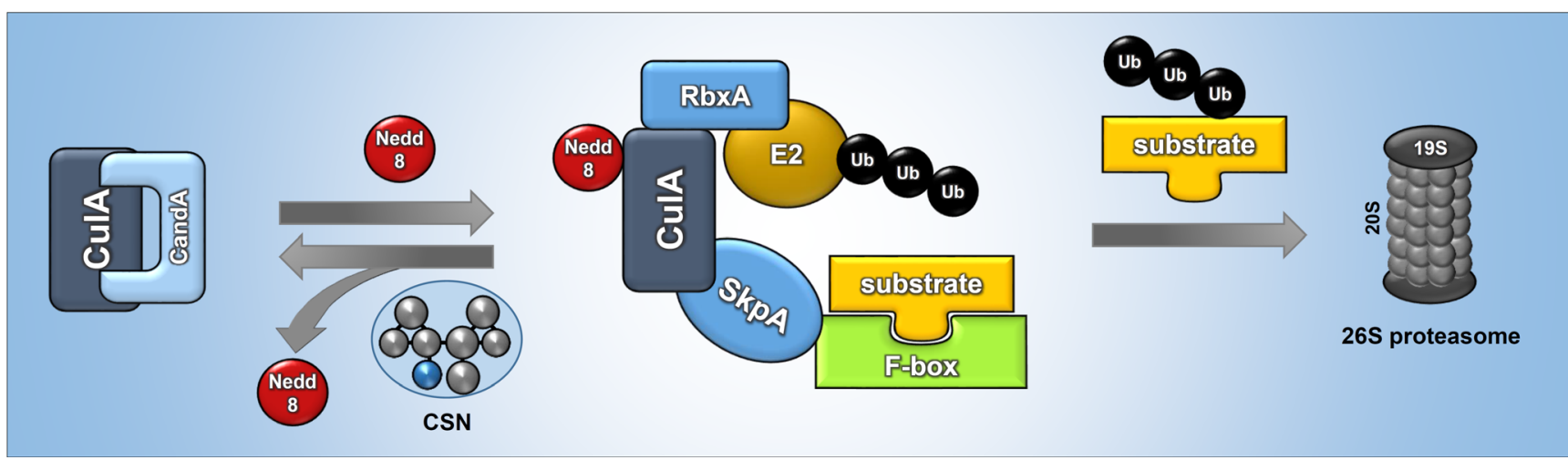

Fig. 2 Action of the COP9 signalosome (CSN) complex (in A. nidulans nomenclature). An SCF complex represents the largest subclass of CULLIN-RING E3 ubiquitin ligases (CRLs). SCF consists of the subunits SkpA, CulA, an F-box protein and the RING protein RbxA. SCF provides the platform for ubiquitination of substrates. The SCF complex is activated by a neddylation/deneddylation cycle. The CSN complex

\section{CSN and fungal development}

The general role of CSN in the control of protein turnover results in numerous pleiotropic effects, which have an impact on processes such as hormone signaling, oxidative stress response, DNA repair, cell cycle, growth, differentiation, light signaling, and secondary metabolism (Dohmann et al. 2008; Nahlik et al. 2010; Wei et al. 1994). CSN is only essential in higher eukaryotes, whereas fungal mutant strains of the corresponding genes are viable but often show a disturbed development. In Saccharomyces cerevisiae, CSN plays a role in adaptation to pheromone signaling, and csn mutants exhibit increased mating efficiency (Maytal-Kivity et al. 2002). In S. pombe, csn mutants are delayed in progression through S-phase and are more sensitive to DNA damage (Mundt et al. 2002). In the filamentous fungus N. crassa, csn mutants are impaired in circadian clock control, show a reduced growth rate, and produce less conidia and aerial hyphae than a wild-type strain (He et al. 2005). Similarly, A. nidulans csn mutants are blind to light and constitutively induce sexual development even under illumination. However, the sexual development is blocked at the level of primordia, and no mature cleistothecia are formed. In contrast, mutant strains in $\operatorname{den} A$, encoding the second deneddylase, which physically interacts with CSN, exhibit an impaired asexual development in A. nidulans (Christmann et al. 2013). Therefore, two interacting deneddylases can contribute to two alternative developmental programs in filamentous fungi. A combined transcriptome, proteome, and metabolome analysis revealed that $15 \%$ of genes are mis-regulated during development in $\Delta c \operatorname{cs} E$, a mutant defective in the deneddylase subunit of CSN (Nahlik et al. 2010). The molecular cause of this developmental defect, which is also linked to a misregulated secondary metabolism (described in the following chapter), correlates with cellular accumulation of with its catalytic subunit CsnE deneddylates the SCF complex, which disassembles. The cullin is bound by CandA. A subsequent neddylation step reassembles the active SCF complex. The F-box protein recruits the substrates and RbxA recruits the ubiquitinated E2 enzyme, which then transfers the ubiquitins to the substrates and targets them for degradation by the $26 \mathrm{~S}$ proteasome

developmentally relevant CULLIN-RING E3 ubiquitin ligases in $\operatorname{csn}$ mutant strains as a consequence of the dysfunctional neddylation-deneddylation cycle (von Zeska Kress et al. 2012).

\section{CSN and secondary metabolism}

The regulation of CSN controlled processes has tremendous influence on secondary metabolism of an organism. In plants, it was found that the biosyntheses of the hormones gibberellic acid in Arabidopsis and jasmonic acid in tomato are reduced in $c s n$ mutants, leading, for example, to diminished resistance against herbivores and pathogens, whereas the biosynthesis of ascorbic acid (vitamin C) in Arabidopsis is elevated (Dohmann et al. 2010; Hind et al. 2011; Wang et al. 2013). In the mold $A$. nidulans, loss of the fifth subunit CsnE, representing the deneddylase subunit, has similar effects on hormone-like psi factor production. The production of the oxylipins 8-hydroxy-9-octadecanoic acid (8-HOE), 8-hydroxy-9,12-octadecadienoic acid (8-HOD), and 10-hydroxy9,12-octadecadienoic acid (10-HOD) was aberrant, explaining the disturbed sexual development (Nahlik et al. 2010). A defined balance of oxylipins is necessary for correct execution of developmental programs (Tsitsigiannis et al. 2004). Additionally, $\Delta \operatorname{csn} E$ accumulates red pigments that were identified as orcinol and the related phenylethers violaceol I and II, cordyol C, and diorcinol. The same compounds were enriched in the candA deletion strains, which further supports the notion that the dysfunctional neddylation-deneddylation cycle of cullin complexes is critical for this phenotype (Helmstaedt et al. 2011).

An untargeted metabolite fingerprinting analysis of $\Delta c s n E$ versus the wild type $A$. nidulans revealed that more than 100 metabolite markers were enriched in the mutant (Nahlik et al. 2010). Additional compounds which normally accumulate in 
A. nidulans are, e.g., precursors of the toxin sterigmatocystin as a member of the aflatoxin polyketide family. In the mutant transcriptome, several gene clusters were upregulated, for which the corresponding products were not yet identified at the time of the analysis. One of them included a gene cluster containing a polyketide synthase (PKS) encoding gene, which is located adjacent to the orsellinic acid gene cluster. The product of the PKS was identified as 2,4-dihydroxy-3-methyl-6-(2-oxopropyl) benzaldehyde (DHMBA, Fig. 1), a polyketide with antibacterial activity (Gerke et al. 2012b). The production of DHMBA illustrated a highly intermingled secondary metabolism. Overexpression of the DHMBA gene cluster abolished the production of another compound, which is only produced by wild-type but not by $\Delta c s n E$ mutant cells. This compound was identified as 3,3-(2,3-dihydroxypropyl) diindole (DHPDI), for which the corresponding biosynthetic genes are not yet identified and presumably located in a different gene cluster of the genome. It is yet unknown how much crosstalk is activated in addition to the mutually exclusive syntheses of either DHPDI or DHMBA. Moreover, the cellular or ecological functions of these metabolites are currently unknown (Gerke et al. 2012b). Further examples of gene clusters that are interdependently regulated are known, and it was shown that these can even be located on different chromosomes (Bergmann et al. 2010; Wiemann et al. 2013).

\section{Velvet-domain protein complexes: linking transcriptional control and chromatin modification}

\section{Velvet complexes and fungal gene regulation}

The velvet domain represents a fungus specific protein-protein interaction domain. Only recently, it could be shown that the velvet domain also represents a DNA-binding domain (Ahmed et al. 2013). Velvet-domain proteins are a specific family of regulators, which are conserved in the fungal kingdom from chytrids to basidiomycetes and are particularly well studied in ascomycetes. Velvet proteins are absent in singlecell yeasts as $S$. cerevisiae or Candida albicans, which coincides with a lack of secondary metabolite gene clusters in these yeasts (Bayram and Braus 2012; Ni and Yu 2007). The name is based on the first mutant strain for a velvet-domain protein, which had been described in A. nidulans as VeA (Velvet A). VeA as the founding member of the velvet family is a light-dependent regulator, which was already introduced to science in 1965 (Kaefer 1965). A mutant strain carried a point mutation in the start codon and produced the truncated version VeA1, missing the first 37 amino acids. The veA1 strain was used over decades as laboratory strain, because it forms more asexual spores than a wild-type strain with an intact VeA protein. In addition, sexual development is retarded and reduced. VeA harbors the conserved velvet domain in the N-terminal region, as well as a nuclear localization signal (NLS) and a nuclear export signal and shuttles between cytoplasm and nucleus (Fig. 3). Additionally, it contains a Cterminally located proline $(\mathrm{P})$, glutamic acid (E), serine (S), and threonine $(\mathrm{T})$ sequence motif (PEST), which suggests that there is a protein half-life control (Rogers et al. 1986). The velvet domain family consists of four members represented by VeA, VelB, VelC, and VosA of $A$. nidulans (and with similar names in other fungi). All of them carry a velvet domain comprising approximately 200 amino acids (Fig. 3).

VelB (Velvet-like protein B) was the second velvet-domain protein described (Bayram et al. 2008a). An interesting feature is that its velvet domain is interrupted by a 99 amino acids insertion (Ahmed et al. 2013). VosA (viability of spores $\underline{A}$ ), another member of the velvet family, was found during a gainof-function genetic screen in A. nidulans and was described as a high-copy repressor of asexual development which couples spore formation and trehalose biosynthesis (Ni and Yu 2007). Besides an N-terminally located velvet domain, VosA comprises a NLS and a C-terminal transcription activation domain (TAD) (Ni and Yu 2007). VosA is a DNA-binding protein that specifically binds to an 11-nucleotide sequence within the promoter regions of more than 1,500 genes in A. nidulans (Ahmed et al. 2013), among them genes involved in asexual development ( $\operatorname{brl} A$, wetA, $\operatorname{vos} A)$ or trehalose biogenesis (tps $A$, treA). Similarly, the VosA and VelB homologs in Histoplasma capsulatum Ryp2 and Ryp3 are DNA-binding proteins (Beyhan et al. 2013). VelC was, until recently, the least studied protein of the velvet family, but, within the last 2 years, it was characterized intensively. It contains one C-terminally located velvet domain with an untypical insertion of a PEST sequence motif which is characteristic for unstable proteins and consists of proline $(\mathrm{P})$, glutamic acid $(\mathrm{E})$, serine $(\mathrm{S})$, and threonine $(\mathrm{T})$ residues (Park et al. 2014).

The 200 amino acids sequence which represents the velvet domain did not reveal significant similarities to other described protein domains. The recently solved crystal structure of the velvet domains of VosA and the heterodimer VosAVelB demonstrated an unexpected similarity to the Rel homology domain (RHD) of the mammalian transcription factor $\mathrm{NF}-\mathrm{KB}$, although the two proteins share only a low amino acid sequence identity of $13.9 \%$ (Ahmed et al. 2013). The family of $\mathrm{NF}-\mathrm{KB}$ responds to external stimuli. RHD containing proteins control development in mammals, inflammation, and the immune response (O'Dea and Hoffmann 2010; Oeckinghaus et al. 2011). The structural similarity between $\mathrm{NF}-\mathrm{KB}$ and the velvet domain could be due to independent convergent evolution or due to a common origin for both proteins. If the second hypothesis is true, a common ancestor would have evolved differently, resulting in the control between development and secondary metabolism in fungi and 
Fig. 3 Domain structure of the four velvet-domain proteins and crystal structure of the velvet domain of VosA in A. nidulans. $V D$, velvet-domain; $N L S$, nuclear localization signal; $N E S$, nuclear export signal; PEST, proline- (P), glutamic acid- $(\mathrm{E})$, serine- $(\mathrm{S})$, and threonine-rich $(\mathrm{T})$ region; $T A D$, transcription activation domain

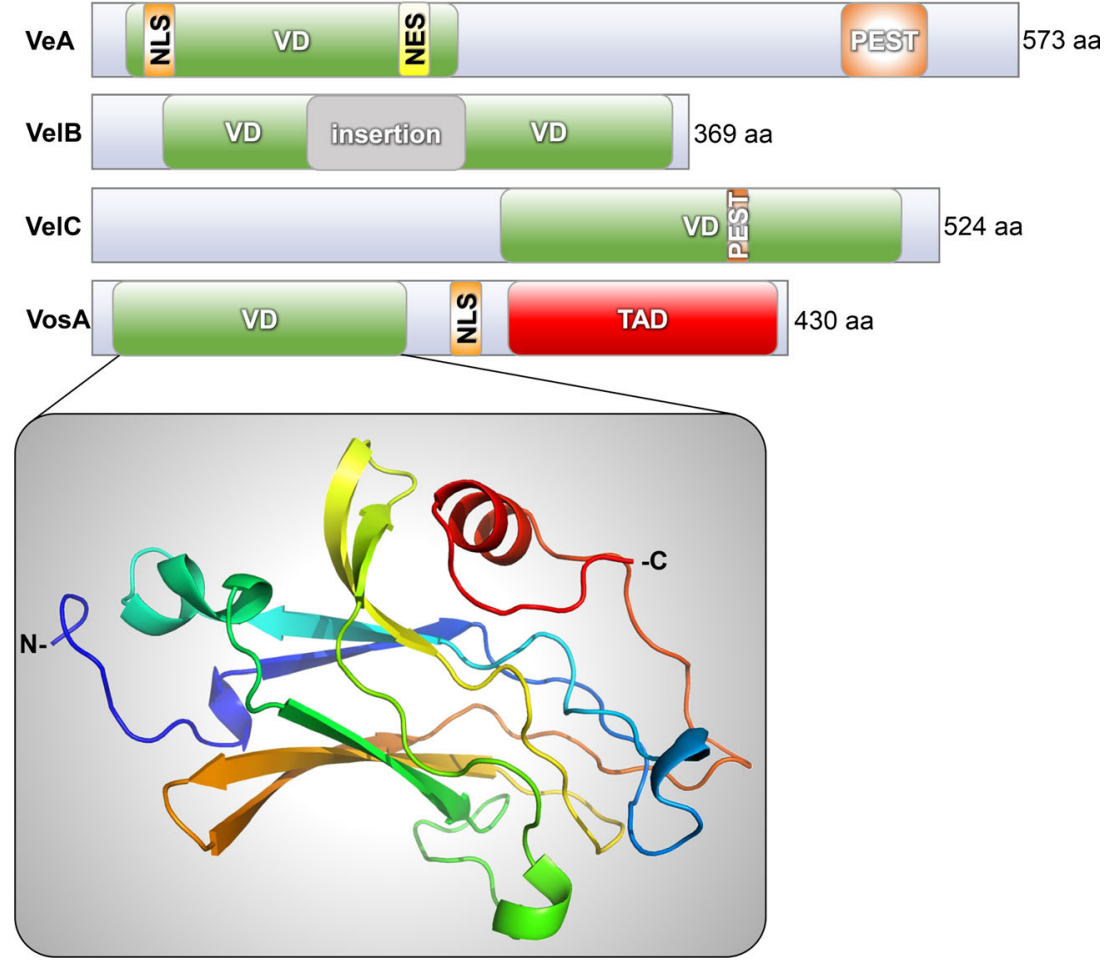

the inflammatory-immune response in mammals (Ahmed et al. 2013).

The velvet-domain proteins are assumed to be a novel group of transcription factors, and the binding sites for members of the family other than VosA have to be elucidated in the future. The velvet-domain proteins can undergo complex formation among themselves, but also with other proteins, leading to different homo- and heterodimers or heterotrimers (Fig. 4). The formation of these complexes in the cell is assumed to be time- and cell-type specific. VeA can form a complex with VelB. During illumination, VeA is primarily located in the cytoplasm. In the absence of light, VeA containing the NLS is transported into the nucleus via the importin $\alpha$ KapA. VelB contains no NLS itself and is channeled into the nucleus via interaction with VeA-KapA. In the nucleus, KapA dissociates, and the dimeric VeA-VelB complex induces sexual development (Bayram et al. 2008a). The VelB-VelB homodimer is formed in the nucleus, and although it is involved in the regulation of asexual development, its exact function and the DNA-binding specificity is unknown. Besides its interaction with VeA and with itself, VelB can also undergo complex formation with VosA. VosA can also form both a homodimer, which represses asexual development, or the heterodimers VosA-VelB and VosAVelC (Ahmed et al. 2013; Beyhan et al. 2013; Park et al. 2012b; Sarikaya Bayram et al. 2010). An interaction of VelC with velvet-domain proteins was not only shown in A. nidulans, but it was also shown in Penicillium chrysogenum and Fusarium oxysporum (Kopke et al. 2013; Lopez-Berges et al. 2013). F. oxysporum VelC interacts with VeA, and P. chrysogenum VelC interacts with VelA and VosA, suggesting the existence of two so far uncharacterized complexes, VelC-VosA and VelC-VeA. Recently, three new VosA interaction partners were found, VoiA, VoiC, and VoiD (VosA-interacting protein), whose function is still unknown (Park et al. 2014).

The first described trimeric velvet complex consists of the two velvet-domain proteins $\mathrm{VeA}$ and $\mathrm{VelB}$ and the putative methyltransferase LaeA (loss of aflR expression $\underline{A}$ ). VeA as the central protein is N-terminally associated with VelB and C-terminally with LaeA. The formation of this trimeric complex is light-dependent. The interaction of the nuclear LaeA with the heterotrimeric $\mathrm{VeA}-\mathrm{VelB}$ in the nucleus links sexual development and secondary metabolism in A. nidulans (Bayram et al. 2008a). LaeA contains a SAM- ( $S$ adenosylmethionine-) binding site, which is essential for its function, and recently, a novel automethylation mechanism was found (Bok et al. 2006; Patananan et al. 2013). VeA also interacts with two additional methyltransferases VipC (velvet interacting protein $\mathrm{C}$ ) and VapB (VipC associated protein $\mathrm{B}$ ). All three methyltransferases LaeA, VipC, and VapB contribute to histone modification and therefore link transcriptional and epigenetic control of gene expression (Sarikaya Bayram et al. 2014). A study on the A. nidulans gene encoding the SAM-synthetase, which catalyzes the formation of the main methyl group donor $S$-adenosylmethionine for 


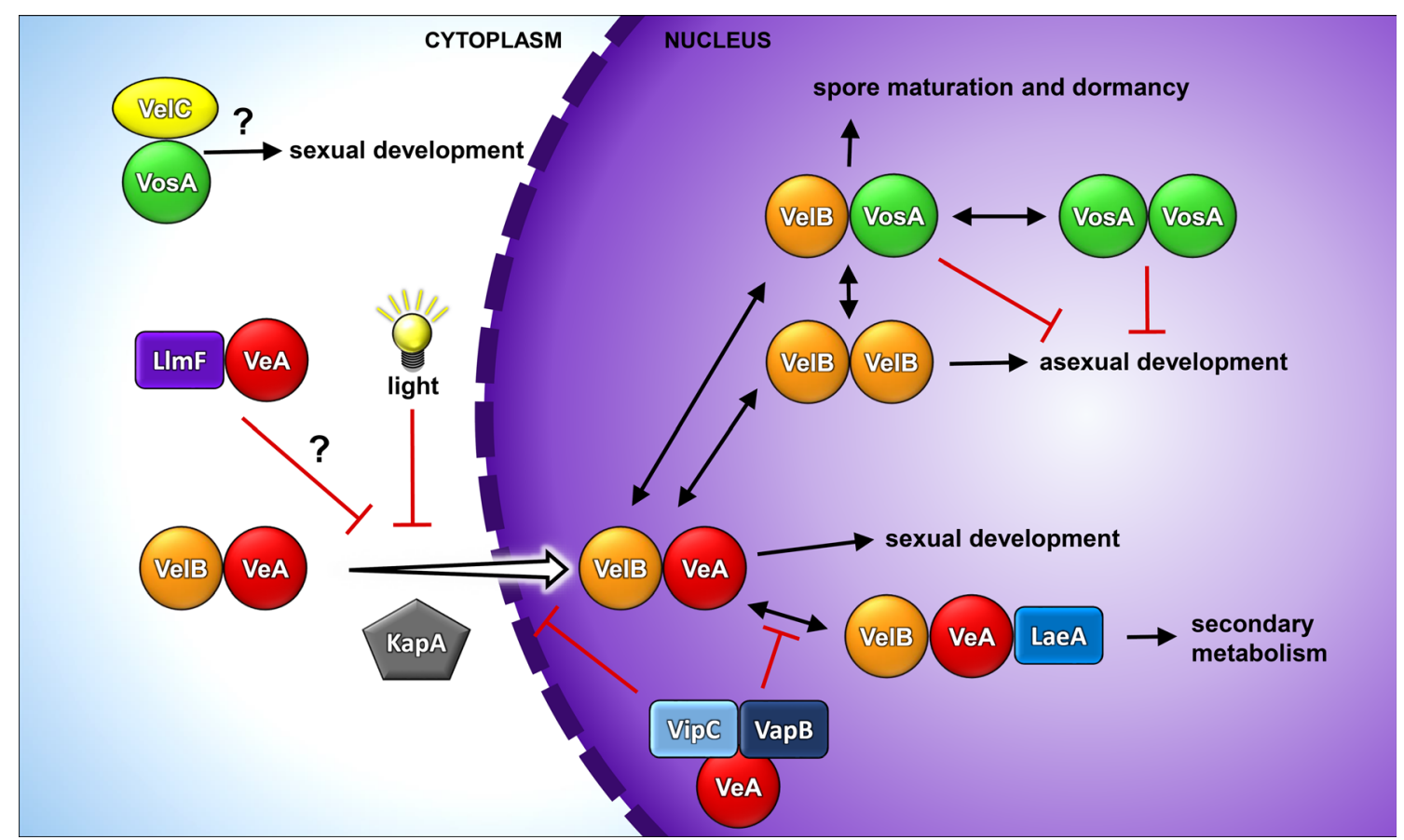

Fig. 4 Interplay of different velvet-domain protein complexes linking fungal development and secondary metabolism. During illumination, $\mathrm{VeA}-\mathrm{VelB}$ are found cytoplasmically. In darkness, VeA-VelB is transported via interaction with KapA into the nucleus, where it initiates sexual development. Via interaction with LaeA, a trimeric complex is formed, which coordinates secondary metabolism. By interaction of VeA with VipC and VapB nuclear import and VelB-VeA-LaeA complex formation is repressed. The VelB-VosA heterodimer is involved in spore maturation and dormancy and represses asexual development. In addition, there are also nuclear VelB and VosA homodimers affecting asexual development. In the cytoplasm, VeA can interact with the methyltransferase LlmF. This complex hinders the nuclear import of VeA. The function of the recently identified cytoplasmic complex VosA-VelC is currently unknown, but it is suggested to be involved in sexual development methyltransferases, also supports an epigenetic link to gene expression in the coordination of fungal development and secondary metabolism (Gerke et al. 2012a).

Velvet complexes and fungal development

Complete loss of the gene for the light regulator VeA leads to the absence of cleistothecia as closed fruiting bodies for sexual ascospores, whereas the overproduction of VeA results in constitutive cleistothecia formation independent of light conditions (Kim et al. 2002). VeA is therefore a positive regulator of sexual development. In Aspergillus parasiticus and A. flavus, lack of the VeA counterpart results in a loss of sclerotia formation. Sclerotia are asexual resting structures of these fungi and are reminiscent of cleistothecia with the exception that neither meiosis nor ascospore formation take place (Calvo et al. 2004; Duran et al. 2007). Deletion of the veA homolog in the plant pathogens Fusarium fujikuroi, Fusarium graminearum, and Fusarium verticillioides results in a decreased number of (micro-)conidia or an increased macroconidia to microconidia ratio, respectively (Li et al. 2006; Merhej et al. 2012; Myung et al. 2009). Similarly, mutations of the corresponding gene of $N$. crassa impair asexual spore formation (Bayram et al. 2008b). These data corroborate a significant role of VeA in different organisms in development, which can be either asexual or sexual differentiation.

Overexpression of velB leads to a twofold increase in the amount of conidia but has no effect on sexual development. Deletion of velB in A. nidulans leads to a loss of cleistothecia formation. Additionally, the mutant strain shows a reduced number of asexual spores as a result of a downregulation of the asexual marker genes $b r l A, a b a A$, and vosA. VelB is part of the two complexes VelB-VosA and VelB-VeA and is therefore involved in the control of both asexual (VelB-VosA) and sexual (VelB-VeA) development (Park et al. 2012b). In the plant pathogen F. fujikuroi, loss of the VelB homologs results in similar phenotypes as loss of VeA homologs with decreased amounts of microconidia (Wiemann et al. 2010). In $F$. graminearum, FgVELB deletion increases conidiation (Jiang et al. 2012). The spores of a vosA or velB deletion mutant in A. nidulans lack trehalose, rapidly lose cytosol, and have a reduced long-term viability and tolerance to oxidative stress or heat. The VosA-VelB heterodimer represses asexual development by negative feedback regulation of the regulatory gene $\operatorname{brl} A$ and positively regulates wetA and $t p s A$, which are involved in spore maturation and trehalose 
biosynthesis. Transcription of both, VosA and VelB, is activated by $\mathrm{AbaA}$ in phialides.

VelC was characterized in A. fumigatus, A. flavus, $P$. chrysogenum, $F$. oxysporum and, recently, also in A. nidulans (Chang et al. 2013; Kopke et al. 2013; LopezBerges et al. 2013; Park et al. 2012a; Park et al. 2014). In F. oxysporum, deletion of velC leads to an increased amount of microconidia and decreased chromatin accessibility (LopezBerges et al. 2013). However, in A. flavus and A. fumigatus, the deletion has no phenotype. In A. nidulans, VelC has an additional role in regulating sexual development (Park et al. 2014). velC is expressed during early sexual development, and deletion of velC decreases the amount of cleistothecia, whereas overexpression induces cleistothecia production. Therefore, VelC is proposed to be an activator of sexual development. The function of the heterodimeric complex VosA-VelC still has to be elucidated (Park et al. 2014). It was proposed that it acts in cleistothecia formation during sexual development. Additionally, the velC deletion increases asexual conidiation, and thus, expression of the asexual regulator genes $\mathrm{brlA}$, $a b a A$, wet $A$, and vosA are upregulated. Due to the fact that the double deletion of velC and $\operatorname{vos} A$ shows the same phenotype as the vosA single deletion, it can be assumed that VosA is epistatic to VelC (Park et al. 2014).

The putative methyltransferase LaeA, which links secondary metabolism to development, also has a major impact on fungal differentiation. LaeA is, together with the two velvetdomain proteins $\mathrm{VeA}$ and VelB, a member of the trimeric velvet complex. In light, the nuclear LaeA reduces the levels of VosA and VelB and with that favors asexual development, whereas, in the dark, the trimeric complex is formed and initiates sexual development. LaeA controls the switch between asexual and sexual development not only in A. nidulans (Bayram et al. 2008a), but also in other ascomycetes like F. fujikuroi and P. chrysogenum (Hoff et al. 2010; Wiemann et al. 2010). In A. nidulans, the laeA mutant is reduced in asexual sporulation and produces smaller fruiting bodies compared with the wild type. This is due to the almost complete lack of globose multinuclear Hülle cells, which have been proposed to be required to nurse the fruiting bodies (Bayram et al. 2008a; Sarikaya Bayram et al. 2010). In A. fumigatus and P. chrysogenum, the conidiophore development is delayed or lacking, respectively (Bok et al. 2005; Hoff et al. 2010). In A. flavus, besides aberrant conidiation, no sclerotia are formed (Chang et al. 2012; Kale et al. 2008), and in F. fujikuroi, the amount of microconidia is reduced (Wiemann et al. 2010). The two additional methyltransferases VipC (velvet interacting protein $\underline{\mathrm{C}}$ ) and $\mathrm{VapB}$ (VipC associated protein $\underline{\mathrm{B}}$ ), which in addition interact with $\mathrm{VeA}$ also have an impact on fungal development in A. nidulans. VipC-VapB either act in the nucleus to promote asexual development or are attached to the plasma membrane in a second trimeric complex with the membrane protein VapA which supports sexual development.
The molecular mechanism which triggers the dissociation of the two methyltransferases from the plasma membrane is currently unknown (Sarikaya Bayram et al. 2014).

Velvet complexes and secondary metabolism

A correlation between development and secondary metabolism was already recognized early in fungal research (reviewed in Calvo et al. 2002), and recently, two secondary metabolites (SMs), dehydroaustinol and diorcinol, were found in signaling the induction of sporulation in A. nidulans (Rodriguez-Urra et al. 2012), and two SM-synthesizing enzymes of $A$. flavus were found to be essential for proper fruiting body development (Forseth et al. 2013). The velvet-domain protein VeA plays an important role in coordination of development with secondary metabolite production. In addition to its function during fruiting body formation, VeA influences secondary metabolism. A veA deletion in A. nidulans abolishes sterigmatocystin production, and the mutant produces red pigments instead (Bayram et al. 2008a; Kato et al. 2003). Similarly, in A. parasiticus and A. flavus, no aflatoxin or aflatoxin precursors are formed in $v e A$ deletion mutants, and it was shown that veA is a global regulator of secondary metabolism (Amare and Keller 2014; Calvo et al. 2004; Duran et al. 2007). Deletion of the veA homolog in the plant pathogens $F$. fujikuroi, $F$. graminearum, and $F$. verticillioides disturbs toxin production. The trichothecene production in $F$. graminearum and the fumonisin and fusarin production in $F$. verticillioides are positively affected by Ve1. In contrast, Vell of F. fujikuroi can as well act as positive (for gibberellins, fumonisins, fusarin $\mathrm{C}$ ) or negative (for bikaverin) regulator of secondary metabolite production (Merhej et al. 2012; Myung et al. 2009; Wiemann et al. 2010). In the biotechnologically relevant fungi $P$. chrysogenum and Acremonium chrysogenum, loss of the VeA homolog results in a reduced production of the antibiotics penicillin and cephalosporin and, in addition, causes defects in development (Dreyer et al. 2007; Hoff et al. 2010).

VelB in A. nidulans is not only a developmental regulator, but loss of the corresponding gene also leads to the accumulation of red pigments, similar to the veA deletion (Bayram et al. 2008a). In F. fujikuroi, loss of the VelB or VeA homologs results in impaired toxin production, and the double deletion of both corresponding genes additionally reduces virulence (Wiemann et al. 2010). In the plant pathogen F. graminearum, FgVELB deletion disturbs pigmentation and reduces pathogenicity (Jiang et al. 2012). In P. chrysogenum, conidiation and the production of penicillin are diminished in a velC deletion mutant (Kopke et al. 2013).

The putative methyltransferase LaeA was originally discovered in A. nidulans as a suppressor of defects in the gene for the specific transcription factor of aflatoxin and sterigmatocystin cluster genes, AflR (Bok and Keller 2004). LaeA is a global regulator of secondary metabolism and controls, amongst others, 
the production of prominent natural products such as aflatoxin, sterigmatocystin, terrequinone, lovastatin, penicillin, gliotoxin, fumonisins, and gibberellins (Baba et al. 2012; Bok and Keller 2004; Bouhired et al. 2007; Kale et al. 2008; Seiboth et al. 2012; Wiemann et al. 2010; Wu et al. 2012).

A multicopy suppressor screen to identify proteins able to restore SM production in the $A$. nidulans laeA deletion mutant revealed additional control proteins (Shaaban et al. 2010; Soukup et al. 2012). RsmA (remediation of secondary $\underline{m}-$ etabolism A) is a basic leucine zipper transcription factor that was found to bind to the aflR promoter and by this to regulate the expression of aflR and sterigmatocystin biosynthetic genes (Yin et al. 2012). According to this study, the overexpression of $r \operatorname{sm} A$ partially restored sterigmatocystin production but not the developmental defects in $\triangle l a e A$ and $\triangle v e A$ (Shaaban et al. 2010). In $A$. fumigatus, the overexpression of $r \operatorname{sm} A$ increased the production of gliotoxin (Sekonyela et al. 2013).

The second protein found in the multicopy suppressor screen was the histone acetyltransferase EsaA (essential

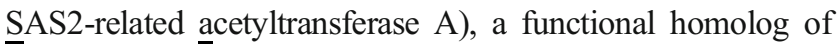
the $S$. cerevisiae Esa1 (Soukup et al. 2012). The overexpression of esaA leads to an increased expression of sterigmatocystin, penicillin, terrequinone, and orsellinic acid gene clusters in A. nidulans, but, for full effect, LaeA is required. In contrast to the yeast Esa1, this effect is not restricted to subtelomeric regions. Like for RsmA, the restoration of the laeA deletion phenotype by EsaA worked for the secondary metabolism defects, but not for developmental defects.

An additional level of complexity in the regulation of secondary metabolism in fungi is the control of cellular compartmentalization. In a reverse genetics screen to identify LaeAlike methyltransferases, $\mathrm{LlmF}$ (LaeA-like methyltransferase $\underline{\mathrm{F}}$ ) was identified in A. nidulans (Palmer et al. 2013) and shortly followed by Llm1 in Cochliobolus heterostrophus (Bi et al. 2013). LlmF is a direct interaction partner of VeA and affects its cellular localization. During overexpression of $l l m F$, VeA is primarily found in the cytoplasm, whereas the deletion of $l \mathrm{~lm} F$ leads to an increased nuclear localization. By this mechanism, L $\operatorname{lmF}$ negatively regulates sterigmatocystin production and, in addition, sexual development (Palmer et al. 2013). Accordingly, in C. heterostrophus, $\mathrm{Llm} 1$ is a negative regulator of T-toxin production (Bi et al. 2013).

\section{Putative potential of fungal mutant strains of genes for CSN and velvet complexes in biotechnology}

\section{Drug discovery}

We urgently need novel drugs to keep up the pace with the rapid increase of microorganisms that develop resistances against established antibiotics. The greatest inventiveness for new active structures is still provided by nature itself. Fungi with its estimated 1.5 million species worldwide (Hawksworth 1991), each supposed to produce several SMs, constitute a reservoir of so far undiscovered chemical structures, which might serve as matrix for the development of new drugs. A simple approach to activate secondary metabolite gene clusters in fungi, which are silenced during vegetative growth and under laboratory conditions, is to produce and take advantage of mutant strains of the two conserved complexes, CSN and velvet.

As described before, the CSN complex regulates the activation of CULLIN-RING E3 ubiquitin ligases (CRLs) and by this, the protein degradation by the proteasome. Loss of a CSN subunit, especially CSN5/CsnE with its catalytic deneddylase activity, leads to mis-regulation of CRLs and to a disturbed protein degradation. This in turn can lead to stabilization of global or specific transcription factors within SM gene clusters, which, under normal conditions, would be degraded rapidly. By using this approach, around 100 metabolite markers were found to be enriched in a $\operatorname{csn} E$ mutant in A. nidulans (Gerke et al. 2012b; Nahlik et al. 2010). This method can be used to identify new SMs, especially for those with silenced biosynthetic gene clusters or to increase the production of special, already known SMs.

Another possibility to activate secondary metabolite gene clusters is the modification of velvet-complex members. The overexpression of the global regulator encoding gene laeA triggers penicillin production in Penicillium and additionally lovastatin production in Aspergillus (Bok and Keller 2004; Kosalkova et al. 2009). Construction of laeA overexpression mutants in different, especially so far metabolically uncharacterized fungi, might lead to the discovery of currently unknown SMs. Furthermore, the formation of the velvet complex is controlled by the subcellular localization of VeA (Palmer et al. 2013). Direct interaction of VeA with the methyltransferase LlmF detains VeA in the cytoplasm and prevents complex formation with VelB and LaeA. In those fungi where this mechanism is conserved, $\operatorname{llm} F$ deletion mutants should permanently induce secondary metabolism and could consequently be used for the discovery of novel SMs.

Besides LaeA and the other methyltransferases which have been reported here, there are additional, promising methyltransferases such as KMT6 of F. graminearum, which are involved in the regulation of secondary metabolism. Loss of KMT6 leads to a lack of H3K27me3 and consequently to a constitutive expression of genes involved in SM biosynthesis (Connolly et al. 2013; Smith et al. 2014).

Toxin contamination

Whereas the utilization of fungal SMs as drugs positively impacts human health, fungi also produce deleterious mycotoxins that can contaminate our food and cause serious 
intoxications and diseases. High levels of aflatoxin, one of the most carcinogenic substances known, can trigger aflatoxicosis (also called Turkey $\mathrm{X}$ disease), which is characterized by acute hepatic necrosis, liver cirrhosis, and carcinomas. The disease was first observed in England in the 1960s, where thousands of poultry died due to aflatoxin contaminated peanut feed (Forgacs and Carll 1962). During an outbreak in Kenya in 2004, more than 300 people suffered from aflatoxicosis with 125 deaths (Lewis et al. 2005). The most common mycotoxigenic fungi that contaminate crops are aflatoxin- and ochratoxin-producing Aspergillus species, ochratoxin- and citrinin-producing Penicillium species and fumonisin- and trichotheceneproducing Fusarium species. The worldwide pre-harvest loss of crops is around $10 \%$ (Normile 2010), whereas the postharvest loss during storage can be even higher. Commercially, around US\$1.2 billion is lost annually due to aflatoxincontaminated crops (Augusto et al. 2013). Different strategies to reduce toxin contamination and improve food safety have been developed. The most promising approach to control aflatoxin contamination is biological control. The use of nontoxigenic $A$. parasiticus and A. flavus strains reduced the preand post-harvest contamination of crops by aflatoxins significantly around $70-90 \%$ by competitive exclusion of the wildtype strains in the field (Yin et al. 2008).

A useful starting point to systematically reduce toxicity of fungi is to target global regulators of secondary metabolism, like the conserved regulator LaeA. The deletion of laeA was shown to reduce the production of aflatoxin and the aflatoxinprecursor sterigmatocystin in different Aspergillus species and the production of trichothecene in Fusarium species (Bok and Keller 2004; Crespo-Sempere et al. 2013; Kale et al. 2008; Lopez-Berges et al. 2013). Additionally, loss of the LaeAinteracting protein VeA diminishes the amount of aflatoxin in Aspergillus and fumonisins in Fusarium (Duran et al. 2007; Myung et al. 2009).

\section{Virulence}

Along with the regulation of secondary metabolism, the proteins involved in velvet complex formation have a tremendous impact on virulence of human and plant pathogenic fungi. The cereal pathogen $F$. graminearum produces a group of mycotoxins called trichothecenes during infection. The deletion of FgLaeA or the velvet genes $\mathrm{FgVeA}$ and $\mathrm{FgVelB}$ diminishes both trichothecene production and virulence in wheat (Lee et al. 2012; Merhej et al. 2012). In F. verticillioides, reduced fumonisins biosynthesis in an FvVE1 mutant goes along with reduced pathogenicity on maize seedlings (Myung et al. 2009), and in F. fujikuroi, single deletions of Ffvell, Ffvel2, and Fflael decrease the amount of gibberellic acid and virulence in rice (Wiemann et al. 2010). T-toxin production is correlated with virulence of the maize pathogen C. heterostrophus. The loss of ChVell and ChLael reduces the T-toxin biosynthesis and, as a consequence, also the virulence of the fungus (Wu et al. 2012). The grey-mold disease inducing Botrytis cinerea loses pathogenicity when bcvell is deleted, and interestingly, already a single nucleotide polymorphism, leading to a truncated protein not able to enter the nucleus, reduces the virulence on different plants (Schumacher et al. 2012). In A. parasiticus, loss of VeA leads to reduced conidiation on peanut seeds (Calvo et al. 2004). Its relative A. flavus exhibits a reduced virulence in maize and peanut seeds, when laeA or veA are deleted (Amaike and Keller 2009). For F. oxysporum, it was shown that velvet complexinduced pathogenicity is not only restricted to plants, but is also an important factor in mammals. The opportunistic human and plant pathogen induces systemic fusariosis in immunocompromised patients and can infect about 100 different crops. An important virulence determinant is beauvericin. Deletion of $v e A, v e l B$, and laeA in F. oxysporum reduces beauvericin synthesis and virulence in tomato and in murine model of fusariosis (Lopez-Berges et al. 2013). In A. fumigatus, the most common inducer of invasive aspergillosis in mammals, loss of LaeA reduces virulence and the amount of pulmonary gliotoxin in mice (Bok et al. 2005). In the human pathogen H. capsulatum, the DNA-binding VosA and VelB homologs Ryp2 and Ryp3 were shown to directly regulate genes involved in pathogenicity (Beyhan et al. 2013). Overall, the velvet complex components, especially LaeA, are promising antifungal drug-targets.

$\mathrm{CSN}$ is also an important complex involved in pathogenicity, but, in contrast to the velvet complex, CSN protects from pathogenic attacks. In plants, it was shown that an intact CSN is necessary for biosynthesis of normal levels of jasmonic acid. In $c s n$-silenced tomato-plants, the amount of jasmonic acid is reduced, and as a result, the plants are less resistant against herbivores and pathogens (Hind et al. 2011). Consequently, it might be interesting to test whether genetically modified plants with induced $c s n$ expression could help to reduce fungal contaminations and to improve crop quality.

\section{Conclusions}

Fungi are industrially important producers of drugs and food additives, but they also have a devastating impact on human and animal health and economy. The current state of increasing resistances against established antibiotics, drastic crop losses, and human mortality rates due to fungal contaminations and infections emphasizes the importance of an exhaustive understanding of the underlying genetic mechanisms in fungi. New bioactive SMs need to be discovered, for which the huge fungal kingdom with its numerous members is well suitable. It is equally important that the molecular mechanisms of virulence and toxin production and the control of the 
biosynthetic pathways will be further illuminated. The CSN and velvet protein complexes act at the interface between secondary metabolism and fungal development. The fact that both complexes are conserved among fungi makes them a perfect target to address issues in agriculture and health, which are highly important for our society. Studies of both complexes in different eukaryotic organisms provided evidence for their conserved functions and showed that both complexes are important players in virulence and production of auxiliary and detrimental SMs.

Acknowledgments This work was supported by the Deutsche Forschungsgemeinschaft (DFG) to GHB (SFB860, SPP1365, BR1502/ 11). We would like to thank Achim Dickmanns and Ralf Ficner for support with the figure of the velvet domain crystal structure.

Open Access This article is distributed under the terms of the Creative Commons Attribution License which permits any use, distribution, and reproduction in any medium, provided the original author(s) and the source are credited.

\section{References}

Ahmed YL, Gerke J, Park HS, Bayram O, Neumann P, Ni M, Dickmanns A, Kim SC, Yu JH, Braus GH, Ficner R (2013) The velvet family of fungal regulators contains a DNA-binding domain structurally similar to NF-kappaB. PLoS Biol 11:e1001750

Amaike S, Keller NP (2009) Distinct roles for VeA and LaeA in development and pathogenesis of Aspergillus flavus. Eukaryot Cell 8: $1051-1060$

Amare MG, Keller NP (2014) Molecular mechanisms of Aspergillus flavus secondary metabolism and development. Fungal Genet Biol 66:11-18

Augusto JA, Akande A, Kaptoge L, Beed F, Olasupo O, Abdoulaye T, Masha K (2013) Ensuring the safety of African food crops. R4D Review 10:11-16.

Baba S, Kinoshita H, Nihira T (2012) Identification and characterization of Penicillium citrinum VeA and LaeA as global regulators for ML236B production. Curr Genet 58:1-11

Bayram O, Braus GH (2012) Coordination of secondary metabolism and development in fungi: the velvet family of regulatory proteins. FEMS Microbiol Rev 36:1-24

Bayram O, Krappmann S, Ni M, Bok JW, Helmstaedt K, Valerius O, Braus-Stromeyer S, Kwon NJ, Keller NP, Yu JH, Braus GH (2008a) $\mathrm{VelB} / \mathrm{VeA} / \mathrm{LaeA}$ complex coordinates light signal with fungal development and secondary metabolism. Science 320:1504-1506

Bayram O, Krappmann S, Seiler S, Vogt N, Braus GH (2008b) Neurospora crassa ve-1 affects asexual conidiation. Fungal Genet Biol 45:127-138

Bergmann S, Funk AN, Scherlach K, Schroeckh V, Shelest E, Horn U, Hertweck C, Brakhage AA (2010) Activation of a silent fungal polyketide biosynthesis pathway through regulatory cross talk with a cryptic nonribosomal peptide synthetase gene cluster. Appl Environ Microbiol 76:8143-8149

Berovic M, Legisa M (2007) Citric acid production. Biotechnol Annu Rev 13:303-343

Beyhan S, Gutierrez M, Voorhies M, Sil A (2013) A temperatureresponsive network links cell shape and virulence traits in a primary fungal pathogen. PLoS Biol 11:e1001614
Bi Q, Wu D, Zhu X, Gillian Turgeon B (2013) Cochliobolus heterostrophus Llm1 - a Lae1-like methyltransferase regulates Ttoxin production, virulence, and development. Fungal Genet Biol 51:21-33

Bok JW, Keller NP (2004) LaeA, a regulator of secondary metabolism in Aspergillus spp. Eukaryot Cell 3:527-535

Bok JW, Balajee SA, Marr KA, Andes D, Nielsen KF, Frisvad JC, Keller NP (2005) LaeA, a regulator of morphogenetic fungal virulence factors. Eukaryot Cell 4:1574-1582

Bok JW, Noordermeer D, Kale SP, Keller NP (2006) Secondary metabolic gene cluster silencing in Aspergillus nidulans. Mol Microbiol 61:1636-1645

Bouhired S, Weber M, Kempf-Sontag A, Keller NP, Hoffmeister D (2007) Accurate prediction of the Aspergillus nidulans terrequinone gene cluster boundaries using the transcriptional regulator LaeA. Fungal Genet Biol 44:1134-1145

Brakhage AA (2013) Regulation of fungal secondary metabolism. Nat Rev Microbiol 11:21-32

Braus GH, Irniger S, Bayram O (2010) Fungal development and the COP9 signalosome. Curr Opin Microbiol 13:672-676

Busch S, Eckert SE, Krappmann S, Braus GH (2003) The COP9 signalosome is an essential regulator of development in the filamentous fungus Aspergillus nidulans. Mol Microbiol 49:717-730

Busch S, Schwier EU, Nahlik K, Bayram O, Helmstaedt K, Draht OW, Krappmann S, Valerius O, Lipscomb WN, Braus GH (2007) An eight-subunit COP9 signalosome with an intact JAMM motif is required for fungal fruit body formation. Proc Natl Acad Sci U S A 104:8089-8094

Calvo AM, Wilson RA, Bok JW, Keller NP (2002) Relationship between secondary metabolism and fungal development. Microbiol Mol Biol Rev 66:447-459

Calvo AM, Bok J, Brooks W, Keller NP (2004) veA is required for toxin and sclerotial production in Aspergillus parasiticus. Appl Environ Microbiol 70:4733-4739

Chang PK, Scharfenstein LL, Ehrlich KC, Wei Q, Bhatnagar D, Ingber BF (2012) Effects of laeA deletion on Aspergillus flavus conidial development and hydrophobicity may contribute to loss of aflatoxin production. Fungal Biol 116:298-307

Chang PK, Scharfenstein LL, Li P, Ehrlich KC (2013) Aspergillus flavus VelB acts distinctly from $\mathrm{VeA}$ in conidiation and may coordinate with FluG to modulate sclerotial production. Fungal Genet Biol 58-59:71-79

Christmann M, Schmaler T, Gordon C, Huang X, Bayram O, Schinke J, Stumpf S, Dubiel W, Braus GH (2013) Control of multicellular development by the physically interacting deneddylases DEN1/DenA and COP9 signalosome. PLoS Genet 9:e1003275

Connolly LR, Smith KM, Freitag M (2013) The Fusarium graminearum histone H3 K27 methyltransferase KMT6 regulates development and expression of secondary metabolite gene clusters. PLoS Genet 9:e1003916

Cooper MA, Shlaes D (2011) Fix the antibiotics pipeline. Nature 472:32

Cope GA, Suh GS, Aravind L, Schwarz SE, Zipursky SL, Koonin EV, Deshaies RJ (2002) Role of predicted metalloprotease motif of Jab1/Csn5 in cleavage of Nedd8 from Cul1. Science 298:608-611

Crespo-Sempere A, Marin S, Sanchis V, Ramos AJ (2013) VeA and LaeA transcriptional factors regulate ochratoxin A biosynthesis in Aspergillus carbonarius. Int J Food Microbiol 166:479-486

Dohmann EM, Levesque MP, De Veylder L, Reichardt I, Jurgens G, Schmid M, Schwechheimer C (2008) The Arabidopsis COP9 signalosome is essential for G2 phase progression and genomic stability. Development 135:2013-2022

Dohmann EM, Nill C, Schwechheimer C (2010) DELLA proteins restrain germination and elongation growth in Arabidopsis thaliana COP9 signalosome mutants. Eur J Cell Biol 89:163-168

Dreyer J, Eichhorn H, Friedlin E, Kurnsteiner H, Kuck U (2007) A homologue of the Aspergillus velvet gene regulates both 
cephalosporin $\mathrm{C}$ biosynthesis and hyphal fragmentation in Acremonium chrysogenum. Appl Environ Microbiol 73:3412-3422

Duda DM, Borg LA, Scott DC, Hunt HW, Hammel M, Schulman BA (2008) Structural insights into NEDD8 activation of cullin-RING ligases: conformational control of conjugation. Cell 134:995-1006

Duran RM, Cary JW, Calvo AM (2007) Production of cyclopiazonic acid, aflatrem, and aflatoxin by Aspergillus flavus is regulated by $v e A$, a gene necessary for sclerotial formation. Appl Microbiol Biotechnol 73:1158-1168

Forgacs J, Carll W (1962) Mycotoxicoses. Adv Vet Sci 7:273-382

Forseth RR, Amaike S, Schwenk D, Affeldt KJ, Hoffmeister D, Schroeder FC, Keller NP (2013) Homologous NRPS-like gene clusters mediate redundant small-molecule biosynthesis in Aspergillus flavus. Angew Chem Int Ed Engl 52:1590-1594

Gerke J, Bayram O, Braus GH (2012a) Fungal $S$-adenosylmethionine synthetase and the control of development and secondary metabolism in Aspergillus nidulans. Fungal Genet Biol 49:443-454

Gerke J, Bayram O, Feussner K, Landesfeind M, Shelest E, Feussner I, Braus GH (2012b) Breaking the silence: protein stabilization uncovers silenced biosynthetic gene clusters in the fungus Aspergillus nidulans. Appl Environ Microbiol 78:8234-8244

Hawksworth DL (1991) The fungal dimension of biodiversity: magnitude, significance, and conservation. Mycol Res 95:641-655

He Q, Cheng P, He Q, Liu Y (2005) The COP9 signalosome regulates the Neurospora circadian clock by controlling the stability of the SCFFWD-1 complex. Genes Dev 19:1518-1531

Helmstaedt K, Schwier EU, Christmann M, Nahlik K, Westermann M, Harting R, Grond S, Busch S, Braus GH (2011) Recruitment of the inhibitor Cand1 to the cullin substrate adaptor site mediates interaction to the neddylation site. Mol Biol Cell 22:153-164

Hertweck C (2009) Hidden biosynthetic treasures brought to light. Nat Chem Biol 5:450-452

Hind SR, Pulliam SE, Veronese P, Shantharaj D, Nazir A, Jacobs NS, Stratmann JW (2011) The COP9 signalosome controls jasmonic acid synthesis and plant responses to herbivory and pathogens. Plant J 65:480-491

Hoff B, Kamerewerd J, Sigl C, Mitterbauer R, Zadra I, Kurnsteiner H, Kuck U (2010) Two components of a velvet-like complex control hyphal morphogenesis, conidiophore development, and penicillin biosynthesis in Penicillium chrysogenum. Eukaryot Cell 9:1236-1250

Inglis DO, Binkley J, Skrzypek MS, Arnaud MB, Cerqueira GC, Shah P, Wymore F, Wortman JR, Sherlock G (2013) Comprehensive annotation of secondary metabolite biosynthetic genes and gene clusters of Aspergillus nidulans, A. fumigatus, A. niger and A. oryzae. BMC Microbiol 13:91

Jiang J, Yun Y, Liu Y, Ma Z (2012) FgVELB is associated with vegetative differentiation, secondary metabolism and virulence in Fusarium graminearum. Fungal Genet Biol 49:653-662

Kaefer E (1965) Origins of translocation in Aspergillus nidulans. Genetics 52:217-232

Kale SP, Milde L, Trapp MK, Frisvad JC, Keller NP, Bok JW (2008) Requirement of LaeA for secondary metabolism and sclerotial production in Aspergillus flavus. Fungal Genet Biol 45:1422-1429

Kato N, Brooks W, Calvo AM (2003) The expression of sterigmatocystin and penicillin genes in Aspergillus nidulans is controlled by veA, a gene required for sexual development. Eukaryot Cell 2:1178-1186

Kim H, Han K, Kim K, Han D, Jahng K, Chae K (2002) The veA gene activates sexual development in Aspergillus nidulans. Fungal Genet Biol 37:72-80

Kobayashi T, Abe K, Asai K, Gomi K, Juvvadi PR, Kato M, Kitamoto K, Takeuchi M, Machida M (2007) Genomics of Aspergillus oryzae. Biosci Biotechnol Biochem 71:646-670

Kopke K, Hoff B, Bloemendal S, Katschorowski A, Kamerewerd J, Kuck U (2013) Members of the Penicillium chrysogenum velvet complex play functionally opposing roles in the regulation of penicillin biosynthesis and conidiation. Eukaryot Cell 12:299-310
Kosalkova K, Garcia-Estrada C, Ullan RV, Godio RP, Feltrer R, Teijeira F, Mauriz E, Martin JF (2009) The global regulator LaeA controls penicillin biosynthesis, pigmentation and sporulation, but not roquefortine $\mathrm{C}$ synthesis in Penicillium chrysogenum. Biochimie 91:214-225

Lee J, Myong K, Kim JE, Kim HK, Yun SH, Lee YW (2012) FgVelB globally regulates sexual reproduction, mycotoxin production and pathogenicity in the cereal pathogen Fusarium graminearum. Microbiology 158:1723-1733

Lewis L, Onsongo M, Njapau H, Schurz-Rogers H, Luber G, Kieszak S, Nyamongo J, Backer L, Dahiye AM, Misore A, DeCock K, Rubin C, Kenya Aflatoxicosis Investigation Group T (2005) Aflatoxin contamination of commercial maize products during an outbreak of acute aflatoxicosis in eastern and central Kenya. Environ Health Perspect 113:1763-1767

Li S, Myung K, Guse D, Donkin B, Proctor RH, Grayburn WS, Calvo AM (2006) FvVE1 regulates filamentous growth, the ratio of microconidia to macroconidia and cell wall formation in Fusarium verticillioides. Mol Microbiol 62:1418-1432

Lim FY, Sanchez JF, Wang CC, Keller NP (2012) Toward awakening cryptic secondary metabolite gene clusters in filamentous fungi. Methods Enzymol 517:303-324

Lo SC, Hannink M (2006) CAND1-mediated substrate adaptor recycling is required for efficient repression of Nrf2 by Keap1. Mol Cell Biol 26:1235-1244

Lopez-Berges MS, Hera C, Sulyok M, Schafer K, Capilla J, Guarro J, Di Pietro A (2013) The velvet complex governs mycotoxin production and virulence of Fusarium oxysporum on plant and mammalian hosts. Mol Microbiol 87:49-65

Maytal-Kivity V, Piran R, Pick E, Hofmann K, Glickman MH (2002) COP9 signalosome components play a role in the mating pheromone response of $S$. cerevisiae. EMBO Rep 3:1215-1221

Merhej J, Urban M, Dufresne M, Hammond-Kosack KE, Richard-Forget F, Barreau C (2012) The velvet gene, FgVel, affects fungal development and positively regulates trichothecene biosynthesis and pathogenicity in Fusarium graminearum. Mol Plant Pathol 13: 363-374

Mundt KE, Liu C, Carr AM (2002) Deletion mutants in COP9/signalosome subunits in fission yeast Schizosaccharomyces pombe display distinct phenotypes. Mol Biol Cell 13:493-502

Myung K, Li S, Butchko RA, Busman M, Proctor RH, Abbas HK, Calvo AM (2009) FvVE1 regulates biosynthesis of the mycotoxins fumonisins and fusarins in Fusarium verticillioides. J Agric Food Chem 57:5089-5094

Nahlik K, Dumkow M, Bayram O, Helmstaedt K, Busch S, Valerius O, Gerke J, Hoppert M, Schwier E, Opitz L, Westermann M, Grond S, Feussner K, Goebel C, Kaever A, Meinicke P, Feussner I, Braus GH (2010) The COP9 signalosome mediates transcriptional and metabolic response to hormones, oxidative stress protection and cell wall rearrangement during fungal development. Mol Microbiol 78:964 979

Newman DJ, Cragg GM (2012) Natural products as sources of new drugs over the 30 years from 1981 to 2010. J Nat Prod 75:311-335

$\mathrm{Ni} \mathrm{M,} \mathrm{Yu} \mathrm{JH} \mathrm{(2007)} \mathrm{A} \mathrm{novel} \mathrm{regulator} \mathrm{couples} \mathrm{sporogenesis} \mathrm{and} \mathrm{trehalose}$ biogenesis in Aspergillus nidulans. PLoS One 2:e970

Normile D (2010) Spoiling for a fight with mold. Science 327:807

O'Dea E, Hoffmann A (2010) The regulatory logic of the NFkappaB signaling system. Cold Spring Harb Perspect Biol 2: a000216

Oeckinghaus A, Hayden MS, Ghosh S (2011) Crosstalk in NF-kappaB signaling pathways. Nat Immunol 12:695-708

Palmer JM, Theisen JM, Duran RM, Grayburn WS, Calvo AM, Keller NP (2013) Secondary metabolism and development is mediated by LlmF control of VeA subcellular localization in Aspergillus nidulans. PLoS Genet 9:e1003193 
Park HS, Bayram O, Braus GH, Kim SC, Yu JH (2012a) Characterization of the velvet regulators in Aspergillus fumigatus. Mol Microbiol 86: 937-953

Park HS, Ni M, Jeong KC, Kim YH, Yu JH (2012b) The role, interaction and regulation of the velvet regulator VelB in Aspergillus nidulans. PLoS One 7:e45935

Park HS, Nam TY, Han KH, Kim SC, Yu JH (2014) VelC positively controls sexual development in Aspergillus nidulans. PLoS One 9: e89883

Patananan AN, Palmer JM, Garvey GS, Keller NP, Clarke SG (2013) A novel automethylation reaction in the Aspergillus nidulans LaeA protein generates $S$-methylmethionine. J Biol Chem 288:1403214045

Rodriguez-Urra AB, Jimenez C, Nieto MI, Rodriguez J, Hayashi H, Ugalde U (2012) Signaling the induction of sporulation involves the interaction of two secondary metabolites in Aspergillus nidulans. ACS Chem Biol 7:599-606

Rogers S, Wells R, Rechsteiner M (1986) Amino acid sequences common to rapidly degraded proteins: the PEST hypothesis. Science 234: 364-368

Sarikaya Bayram O, Bayram O, Valerius O, Park HS, Irniger S, Gerke J, Ni M, Han KH, Yu JH, Braus GH (2010) LaeA control of velvet family regulatory proteins for light-dependent development and fungal cell-type specificity. PLoS Genet 6:e1001226

Sarikaya Bayram O, Bayram O, Feussner K, Kim JH, Kim HS, Kaever A, Feussner I, Chae KS, Han DM, Han KH, Braus GH (2014) The membrane-bound VapA-VipC-VapB methyltransferase complex guides signal transduction for epigenetic and transcriptional control of fungal development. Dev Cell 29:1-15

Schmidt MW, McQuary PR, Wee S, Hofmann K, Wolf DA (2009) F-boxdirected CRL complex assembly and regulation by the CSN and CAND1. Mol Cell 35:586-597

Schumacher J, Pradier JM, Simon A, Traeger S, Moraga J, Collado IG, Viaud M, Tudzynski B (2012) Natural variation in the VELVET gene bcvell affects virulence and light-dependent differentiation in Botrytis cinerea. PLoS One 7:e47840

Seiboth B, Karimi RA, Phatale PA, Linke R, Hartl L, Sauer DG, Smith KM, Baker SE, Freitag M, Kubicek CP (2012) The putative protein methyltransferase LAE1 controls cellulase gene expression in Trichoderma reesei. Mol Microbiol 84:1150-1164

Sekonyela R, Palmer JM, Bok JW, Jain S, Berthier E, Forseth R, Schroeder F, Keller NP (2013) RsmA regulates Aspergillus fumigatus gliotoxin cluster metabolites including cyclo (L-Phe-LSer), a potential new diagnostic marker for invasive aspergillosis. PLoS One 8:e62591

Shaaban MI, Bok JW, Lauer C, Keller NP (2010) Suppressor mutagenesis identifies a velvet complex remediator of Aspergillus nidulans secondary metabolism. Eukaryot Cell 9:1816-1824
Singh OV, Kumar R (2007) Biotechnological production of gluconic acid: future implications. Appl Microbiol Biotechnol 75:713-722

Smith KM, Gautschi JT, Freitag M (2014) Decoding the cryptic genomes of fungi: the promise of novel antibiotics. Future Microbiol 9:265-268

Soukup AA, Chiang YM, Bok JW, Reyes-Dominguez Y, Oakley BR, Wang CC, Strauss J, Keller NP (2012) Overexpression of the Aspergillus nidulans histone 4 acetyltransferase EsaA increases activation of secondary metabolite production. Mol Microbiol 86: 314-330

Tsitsigiannis DI, Zarnowski R, Keller NP (2004) The lipid body protein, PpoA, coordinates sexual and asexual sporulation in Aspergillus nidulans. J Biol Chem 279:11344-11353

von Zeska Kress MR, Harting R, Bayram O, Christmann M, Irmer H, Valerius O, Schinke J, Goldman GH, Braus GH (2012) The COP9 signalosome counteracts the accumulation of cullin SCF ubiquitin E3 RING ligases during fungal development. Mol Microbiol 83: $1162-1177$

Wang J, Yu Y, Zhang Z, Quan R, Zhang H, Ma L, Deng XW, Huang R (2013) Arabidopsis CSN5B interacts with VTC1 and modulates ascorbic acid synthesis. Plant Cell 25:625-636

Wei N, Chamovitz DA, Deng XW (1994) Arabidopsis COP9 is a component of a novel signaling complex mediating light control of development. Cell 78:117-124

Wei N, Serino G, Deng XW (2008) The COP9 signalosome: more than a protease. Trends Biochem Sci 33:592-600

Went FAFC (1895) Monascus purpureus le champignon de l'angquac une nouvelle thelebolee. Ann Sc Nat Bot 8:1-17

Wiemann P, Brown DW, Kleigrewe K, Bok JW, Keller NP, Humpf HU, Tudzynski B (2010) FfVell and FfLae1, components of a velvet-like complex in Fusarium fujikuroi, affect differentiation, secondary metabolism and virulence. Mol Microbiol 77:972-994

Wiemann P, Guo CJ, Palmer JM, Sekonyela R, Wang CC, Keller NP (2013) Prototype of an intertwined secondary-metabolite supercluster. Proc Natl Acad Sci U S A 110:17065-17070

Wu JT, Lin HC, Hu YC, Chien CT (2005) Neddylation and deneddylation regulate $\mathrm{Cul} 1$ and $\mathrm{Cul} 3$ protein accumulation. Nat Cell Biol 7:1014-1020

Wu D, Oide S, Zhang N, Choi MY, Turgeon BG (2012) ChLael and ChVel1 regulate T-toxin production, virulence, oxidative stress response, and development of the maize pathogen Cochliobolus heterostrophus. PLoS Pathog 8:e1002542

Yin YN, Yan LY, Jiang JH, Ma ZH (2008) Biological control of aflatoxin contamination of crops. J Zhejiang Univ Sci B 9:787-792

Yin WB, Amaike S, Wohlbach DJ, Gasch AP, Chiang YM, Wang CC, Bok JW, Rohlfs M, Keller NP (2012) An Aspergillus nidulans bZIP response pathway hardwired for defensive secondary metabolism operates through aflR. Mol Microbiol 83:1024-1034 